\title{
Spatial dimensions of the electron diffusion region in anti-parallel magnetic reconnection
}

\author{
Takuma Nakamura ${ }^{1}$, Rumi Nakamura ${ }^{1}$, and Hiroshi Haseagwa ${ }^{2}$ \\ ${ }^{1}$ Space Research Institute, Austrian Academy of Sciences, Graz 8042, Austria \\ ${ }^{2}$ Institute of Space and Astronautical Science, JAXA, Sagamihara, Japan \\ Correspondence to: Takuma Nakamura (takuma.nakamura@oeaw.ac.at)
}

Received: 30 October 2015 - Revised: 3 February 2016 - Accepted: 14 March 2016 - Published: 24 March 2016

\begin{abstract}
Spatial dimensions of the detailed structures of the electron diffusion region in anti-parallel magnetic reconnection were analyzed based on two-dimensional fully kinetic particle-in-cell simulations. The electron diffusion region in this study is defined as the region where the positive reconnection electric field is sustained by the electron inertial and non-gyrotropic pressure components. Past kinetic studies demonstrated that the dimensions of the whole electron diffusion region and the inner non-gyrotropic region are scaled by the electron inertial length $d_{\mathrm{e}}$ and the width of the electron meandering motion, respectively. In this study, we successfully obtained more precise scalings of the dimensions of these two regions than the previous studies by performing simulations with sufficiently small grid spacing $\left(1 / 16-1 / 8 d_{\mathrm{e}}\right)$ and a sufficient number of particles (800 particles cell ${ }^{-1}$ on average) under different conditions changing the ion-to-electron mass ratio, the background density and the electron $\beta_{\mathrm{e}}$ (temperature). The obtained scalings are adequately supported by some theories considering spatial variations of field and plasma parameters within the diffusion region. In the reconnection inflow direction, the dimensions of both regions are proportional to $d_{\mathrm{e}}$ based on the background density. Both dimensions also depend on $\beta_{\mathrm{e}}$ based on the background values, but the dependence in the inner region $(\sim 0.375$ th power $)$ is larger than the whole region (0.125th power) reflecting the orbits of meandering and accelerated electrons within the inner region. In the outflow direction, almost only the non-gyrotropic component sustains the positive reconnection electric field. The dimension of this single-scale diffusion region is proportional to the ionelectron hybrid inertial length $\left(d_{\mathrm{i}} d_{\mathrm{e}}\right)^{1 / 2}$ based on the background density and weakly depends on the background $\beta_{\mathrm{e}}$ with the 0.25 th power. These firm scalings allow us to predict
\end{abstract}

observable dimensions in real space which are indeed in reasonable agreement with past in situ spacecraft observations in the Earth's magnetotail and have important implications for future observations with higher resolutions such as the NASA Magnetospheric Multiscale (MMS) mission.

Keywords. Magnetospheric physics (magnetotail) - space plasma physics (magnetic reconnection; numerical simulation studies)

\section{Introduction}

Magnetic reconnection is one of the most important energy transfer processes in space and laboratory plasmas, which converts magnetic energy to kinetic energy by changing the magnetic field topology (e.g., Birn and Priest, 2007; Yamada et al., 2010; Paschmann et al., 2013; Treumann and Baumjohann, 2015). The topology change in the reconnection process takes place in a small-scale region called the diffusion region where plasmas are decoupled from the magnetic field. The diffusion region in collisionless plasmas is known to have a multi-scale structure based on ion and electron scales, which can be described by the extended Ohm's law derived from the electron momentum equation (e.g., Kuznetsova et al., 1998; Pritchett, 2001),

$$
\begin{aligned}
\boldsymbol{E} & =-\frac{1}{c} \boldsymbol{U}_{\mathrm{e}} \times \boldsymbol{B}-\frac{\nabla \cdot \boldsymbol{P}_{\mathrm{e}}}{e n}-\frac{m_{\mathrm{e}}}{e} \frac{\mathrm{d} \boldsymbol{U}_{\mathrm{e}}}{\mathrm{d} t} \\
& =-\frac{1}{c} \boldsymbol{U}_{\boldsymbol{i}} \times \boldsymbol{B}+\frac{\boldsymbol{J} \times \boldsymbol{B} / c}{e n}-\frac{\nabla \cdot \boldsymbol{P}_{\mathrm{e}}}{e n}-\frac{m_{\mathrm{e}}}{e} \frac{\mathrm{d} \boldsymbol{U}_{\mathrm{e}}}{\mathrm{d} t},
\end{aligned}
$$

where $\boldsymbol{J}$ is the current density, $\boldsymbol{P}_{\mathrm{e}}$ is the electron pressure tensor, $n$ is the number density and $\boldsymbol{U}_{\mathrm{i}}$ and $\boldsymbol{U}_{\mathrm{e}}$ are the ion and electron bulk flow velocities, respectively. Considering a 
two-dimensional situation in which reconnection develops in the $x-z$ plane, reconnection is sustained by the $y$ component of the electric field $E_{y}$ and the multi-scale structure of the diffusion region can be described by the $y$ component of each term in Eq. (1),

$$
\begin{aligned}
& E_{y}= \underbrace{-\left(\boldsymbol{U}_{\mathrm{i}} \times \boldsymbol{B}\right)_{y}+(\boldsymbol{J} \times \boldsymbol{B} / \text { cen })_{y}}_{\left.E_{y}^{\mathrm{VB}}\right|_{\text {ion }}} \\
& E_{y}^{\mathrm{HB}}=-\left(\boldsymbol{U}_{\mathrm{e}} \times \boldsymbol{B}\right)_{y} \\
& \underbrace{-\frac{1}{\mathrm{en}}\left(\frac{\partial P_{\mathrm{e} x y}}{\partial x}+\frac{\partial P_{\mathrm{e} y z}}{\partial z}\right)}_{E_{y}^{\mathrm{NG}}} \\
& \underbrace{-\frac{m_{\mathrm{e}}}{e}\left[\frac{\partial \boldsymbol{U}_{\mathrm{e}}}{\partial t}+\left(\boldsymbol{U}_{\mathrm{e}} \cdot \nabla\right) \boldsymbol{U}_{\mathrm{e}}\right]_{y}}_{E_{y}^{\mathrm{EI}} .}
\end{aligned}
$$

The convection term $\left.E_{y}^{\mathrm{VB}}\right|_{\text {ion }}$ is dominant outside the diffusion region where electrons and ions move together. In the outer diffusion region where ions are decoupled from electrons, the Hall term $E_{y}^{\mathrm{Hall}}$ is not negligible and $E_{y}^{\mathrm{VB}}$ is dominant. In the inner diffusion region, electron inertial and kinetic (non-gyrotropic pressure) effects (i.e., $E_{y}^{\mathrm{EI}}$ and $E_{y}^{\mathrm{NG}}$ ) play central roles to dissipate the magnetic energy. In this region, $E_{y}^{\mathrm{rec}}=\left(\boldsymbol{E}+\boldsymbol{U}_{\mathrm{e}} \times \boldsymbol{B}\right)_{y} \sim E_{y}^{\mathrm{NG}}+E_{y}^{\mathrm{EI}}$, which is sometimes called the reconnection electric field, is dominant. In this paper, the region where $E_{y}^{\mathrm{rec}}>0$ is defined as the electron diffusion region.

Past numerical studies considering the finite-mass electron fluid showed that the dimension of the whole electron diffusion region in the inflow direction ( $\mathrm{z}$ direction in this paper) is controlled by the electron inertial term $E_{y}^{\mathrm{EI}}$ and scaled by the electron inertial length $d_{\mathrm{e}}$ (e.g., Shay et al., 2001). Fully kinetic simulations further demonstrated that near the center of the diffusion region (the reconnection X-point), $E_{y}^{\mathrm{EI}}$ is negligible and instead the non-gyrotropic term $E_{y}^{\mathrm{NG}}$ is dominant (e.g., Hesse and Winske, 1998; Pritchett, 2001). This non-gyrotropic pressure component is known to result from the electron meandering motion whose width can be described by a hybrid of the electron gyroradius and the magnetic field gradient scale $\left(\rho_{\mathrm{e}} L_{B}\right)^{1 / 2}$ (e.g., Horiuchi and Sato, 1994; Kuznetsova et al., 1998; Hesse et al., 1999; Dorfman et al., 2008). However, these expressions are not enough to predict precise dimensions of the whole diffusion region and the inner non-gyrotropic region, since the inertial length $d_{\mathrm{e}}$ and the meandering width can vary within the diffusion region depending on the spatial variations of associated field and plasma parameters. For example, although Shay et al. (2001) obtained the scaling of the dimension of the diffusion region $\left(\propto d_{\mathrm{e}}\right)$ by surveying the dependence on the ion-to-electron mass ratio, $d_{\mathrm{e}}$ can also vary depending on the density variation between the background and the sheet center. The meandering width can also be affected by the non-uniform tem- perature due to a local electron acceleration within the inner region.

In this paper, we performed a series of fully kinetic particle-in-cell simulations changing the ion-to-electron mass ratio, the background plasma density and the electron temperature to measure the spatial dimensions of the whole diffusion region and the inner non-gyrotropic region under different conditions. The simulations with sufficiently high resolutions and large number of particles allowed us to obtain clear scaling laws of the dimensions of both regions with sufficiently small errors. We here obtained the scalings separately in the reconnection inflow and outflow directions. The obtained scalings in both directions can successfully be explained by some theories considering the spatial variations of the associated parameters including the electron density and temperature within the diffusion region, indicating the adequacy of the scalings. This is the first study that obtains such firm scalings of the dimensions of both regions, which predict precise dimensions in real space and provide important implication to spacecraft observations including the NASA Magnetospheric Multiscale (MMS) mission.

This paper is organized as follows. In Sect. 2, we present the simulation setup employed in this paper. Section 3 contains the overview of the simulation results (Sect. 3.1) and the detailed analyses and theories on the scaling in each (inflow/outflow) direction (Sects. 3.2-3.4). In Sect. 4, we summarize the results and discuss the implications for spacecraft observations.

\section{Simulation setup}

We employ the fully kinetic particle-in-cell (PIC) code VPIC (cf., Bowers et al., 2008). The simulations shown in this paper are $2-1 / 2$ dimensional in the $x-z$ plane. The initial parameters are similar to the ones employed in the Geospace Environmental Modeling (GEM) magnetic reconnection challenge (Birn et al., 2001; Pritchett, 2001). The initial magnetic field and the corresponding number density profiles are set up as $B_{x}(z)=B_{x 0} \tanh \left(z / D_{0}\right)$ (Harris sheet) and $n_{i 0}(z)=n_{e 0}(z)=n_{0} \operatorname{sech}^{2}\left(z / D_{0}\right)+n_{0 \infty}$, where $D_{0}$ is the half-thickness of the initial current sheet, and $n_{0}$ and $n_{0 \infty}$ are the Harris and background density components, respectively. $D_{0}$ is set to be $0.5 d_{\mathrm{i} 0}$, where $d_{\mathrm{i} 0}$ is the ion inertial length based on $n_{0} . \beta_{0}=\beta_{\mathrm{i} 0}+\beta_{\mathrm{e} 0}$ based on $B_{0}$ and $n_{0}$ is 1.0. The ratio between the electron plasma frequency and the gyrofrequency $\omega_{\mathrm{pe}} / \Omega_{\mathrm{e}}=2.0$. The system size based on $d_{\mathrm{i} 0}$ is fixed as $L_{x} \times L_{z}=25.6 d_{\mathrm{i} 0} \times 12.8 d_{\mathrm{i} 0}$. The boundary conditions are periodic along the $x$ direction, with conducting walls along the $z$ direction. An initial magnetic field perturbation is added according to $\delta \boldsymbol{B}=\boldsymbol{z} \times \nabla \psi$, where $\psi(x, z)=$ $0.1 \cos \left(2 \pi x / L_{x}\right) \cos \left(\pi x / L_{z}\right)$.

In this paper, we survey the grid spacing, the averaged number of particles per cell $N_{\mathrm{p}} /$ cell, the ion-to-electron mass 
Table 1. System size, $N_{\mathrm{p}} /$ cell, $m_{\mathrm{i}} / m_{\mathrm{e}}, T_{\mathrm{i} 0} / T_{\mathrm{e} 0}, \beta_{\mathrm{e} 0}$ and $\mathrm{d} x$ normalized by $d_{\mathrm{e} 0}\left(d_{\mathrm{e} \infty}\right)$ and $\rho_{\mathrm{e} 0}$ for each run shown in this paper.

\begin{tabular}{lcccccccc}
\hline Run & System size (grids) & $N_{\mathrm{p}} /$ cell & $n_{\mathrm{e} \infty} / n_{0}$ & $m_{\mathrm{i}} / m_{\mathrm{e}}$ & $T_{\mathrm{i} 0} / T_{\mathrm{e} 0}$ & $\beta_{\mathrm{e} 0}$ & $\mathrm{~d} x / d_{\mathrm{e} 0}\left(\mathrm{~d} x / d_{\mathrm{e} \infty}\right)$ & $\mathrm{d} x / \rho_{\mathrm{e} 0}$ \\
\hline 1 & $1024 \times 512$ & 100 & 0.2 & 25 & 5 & 0.167 & $0.125(0.056)$ & 0.433 \\
2 & $1024 \times 512$ & 800 & 0.2 & 25 & 5 & 0.167 & $0.125(0.056)$ & 0.433 \\
3 & $2048 \times 1024$ & 800 & 0.2 & 25 & 5 & 0.167 & $0.063(0.028)$ & 0.217 \\
4 & $1024 \times 512$ & 800 & 0.5 & 25 & 5 & 0.167 & $0.125(0.088)$ & 0.433 \\
5 & $2048 \times 1024$ & 800 & 0.2 & 100 & 5 & 0.167 & $0.125(0.056)$ & 0.433 \\
6 & $4096 \times 2048$ & 800 & 0.2 & 400 & 5 & 0.167 & $0.125(0.056)$ & 0.433 \\
7 & $2048 \times 1024$ & 800 & 0.2 & 25 & 2 & 0.333 & $0.063(0.028)$ & 0.153 \\
8 & $2048 \times 1024$ & 800 & 0.2 & 25 & 11 & 0.083 & $0.063(0.028)$ & 0.306 \\
\hline
\end{tabular}

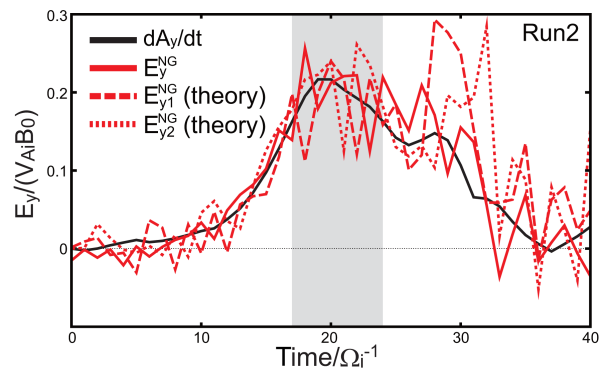

Figure 1. Time evolution of the reconnection rate $\left(\mathrm{d} A_{y} / \mathrm{d} t\right)$, $E_{y}^{\mathrm{NG}}$ and the approximated $E_{y 1}^{\mathrm{NG}}=\frac{\sqrt{2 m_{\mathrm{e}} T_{\mathrm{e} x x}}}{e} U_{\mathrm{e} x}^{\prime}$ and $E_{y 2}^{\mathrm{NG}}=$ $\frac{\sqrt{2 m_{\mathrm{e}} T_{\mathrm{e} z z}}}{e} U_{\mathrm{e} z}^{\prime}$ measured at the X-point. The shaded interval indicates the quasi-steady phase which is defined as the time when $\left|\mathrm{d} A_{y} / \mathrm{d} t\right|>0.75\left|\mathrm{~d} A_{y} / \mathrm{d} t\right|_{\max }$ where $\left|\mathrm{d} A_{y} / \mathrm{d} t\right|_{\max }$ is the maximum value of the reconnection rate.

ratio $m_{\mathrm{i}} / m_{\mathrm{e}}$, the ion-to-electron temperature ratio $T_{\mathrm{i} 0} / T_{\mathrm{e} 0}$ and $n_{0 \infty}$ as listed in Table 1 .

\section{Results}

\subsection{Overview}

The black solid line in Fig. 1 shows the time evolution of the reconnection rate $\left(\mathrm{d} A_{y} / \mathrm{d} t\right)$ for Run 2. The rate rapidly increases after $t=10 \Omega_{i}^{-1}$ and saturates (reconnection goes to a quasi-steady phase) around $t=20 \Omega_{i}^{-1}$. Figure $2 \mathrm{a}-\mathrm{c}$ shows color contours of the reconnection electric field $E_{y}^{\text {rec }}=\left(\boldsymbol{E}+\boldsymbol{U}_{\mathrm{e}} \times \boldsymbol{B}\right)_{y}$, the electron inertial term $E_{y}^{\mathrm{EI}}=\frac{m_{\mathrm{e}}}{e}\left[\left(\boldsymbol{U}_{\mathrm{e}} \cdot \nabla\right) \boldsymbol{U}_{\mathrm{e}}\right]_{y}$ and the non-gyrotropic term $E_{y}^{\mathrm{NG}}=$ $-\frac{1}{\text { en }}$ e $\left(\partial P_{\text {exy }} / \partial x+\partial P_{\text {eyz }} / \partial z\right)$ for Run $2\left(800\right.$ particles cell $\left.^{-1}\right)$ at $t=20 \Omega_{i}^{-1}$ without any smoothing techniques. Figure $2 \mathrm{~d}$ shows $E_{y}^{\mathrm{NG}}$ at $t=20 \Omega_{i}^{-1}$ for Run 1 (100 particles cell $\left.{ }^{-1}\right)$. The thermal noise due to the PIC methods is generally reduced by increasing the number of particles per cell. We have confirmed that runs with more than 500 particles cell $^{-1}$ allow us to see sufficiently clear structures of $E_{y}^{\text {rec }}, E_{y}^{\mathrm{EI}}$ and $E_{y}^{\mathrm{NG}}$ (compare Fig. 2c and d). A similar result to Run 2

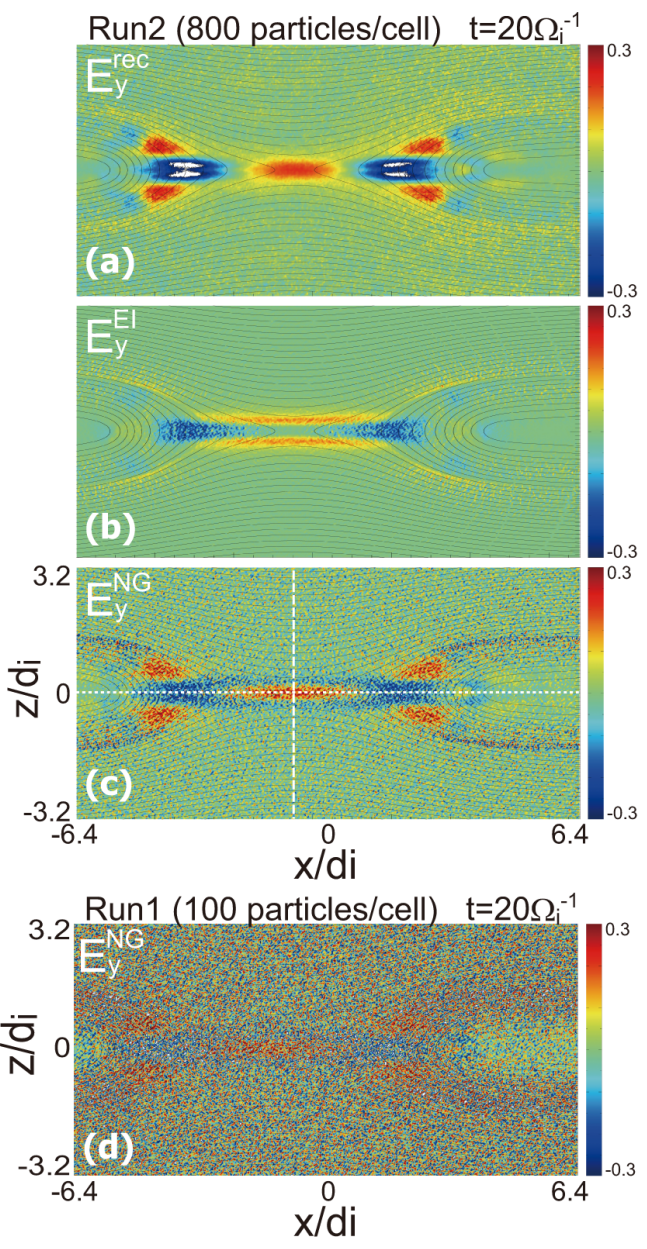

Figure 2. Color contours of $(\mathbf{a}-\mathbf{c}) E_{y}^{\mathrm{rec}}=\left(\boldsymbol{E}+\boldsymbol{U}_{\mathrm{e}} \times \boldsymbol{B}\right)_{y}, E_{y}^{\mathrm{EI}}=$

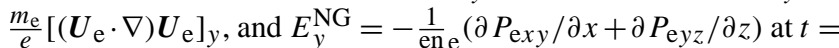
$20 \Omega_{i}^{-1}$ for Run 2 (800 particles cell ${ }^{-1}$ ) and (d) $E_{y}^{\mathrm{NG}}$ for Run 1 (100 particles cell $\left.{ }^{-1}\right)$.

(i.e., clear $E_{y}$ structures without smoothing) was obtained in Run 3 where the spatial resolution is twice as high as that in Run 2 (not shown here), indicating that the particle number and the grid spacing for Run 2 (800 particles cell $^{-1}$ and $\mathrm{d} x=8 d_{\mathrm{e} 0} \sim 2.3 \rho_{\mathrm{e} 0}$, where $\rho_{\mathrm{e} 0}$ is the electron gyrora- 

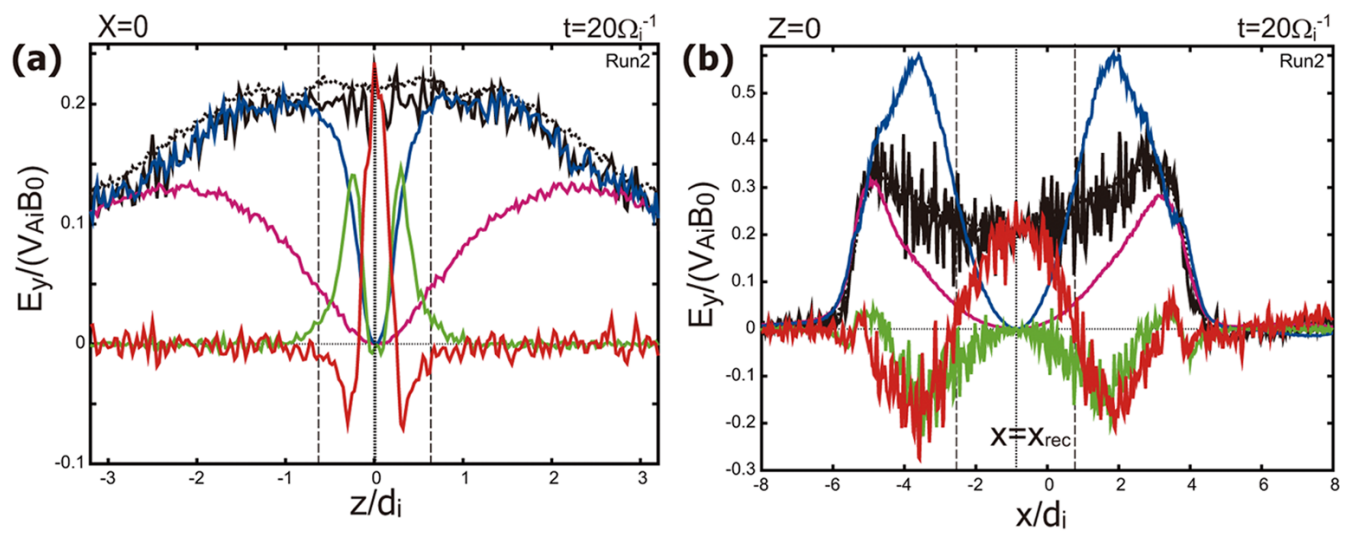

Figure 3. Cuts from the white dashed $\left(X=x-x_{\text {rec }}=0\right)$ and dotted $(z=0)$ lines in Fig. 2c which cross the X-point of $E_{y}^{\mathrm{NG}}$ (red), $E_{y}^{\mathrm{EI}}(\mathrm{green})$, $E_{y}^{\mathrm{VB}}=\left(-\boldsymbol{U}_{\mathrm{e}} \times \boldsymbol{B}\right)_{y}$ (blue), $E_{y}^{\mathrm{tot}}=E_{y}^{\mathrm{NG}}+E_{y}^{\mathrm{EI}}+E_{y}^{\mathrm{VB}}$ (black solid), $E_{y}$ (black dashed) and $\left.E_{y}^{\mathrm{VB}}\right|_{\text {ion }}=\left(-\boldsymbol{U}_{\boldsymbol{i}} \times \boldsymbol{B}\right)_{y}$ (magenta). The plots are smoothed by averaging the data over $|X| \leq 1.75 d_{\mathrm{e} 0}$ for (a) and $|z| \leq 0.25 d_{\mathrm{e} 0}$ for (b). Vertical dotted lines show the locations of the $\mathrm{X}$-point, and vertical dashed lines in (a) and (b) show the locations of $|z|=L_{z}^{\mathrm{Df}}$ and $|X|=L_{x}^{\mathrm{Df}}$, respectively.

dius based on $B_{0}$ ) would be enough to examine the structure within the electron diffusion region. In this paper, using runs with 800 particles cell $^{-1}$ and similar (or higher) spacial resolutions (Runs 2-8 in Table 1), we examined the scaling of the spatial dimensions of the electron diffusion region.

Figure 3 shows cuts from the white dashed $\left(X=x-x_{\text {rec }}\right)$ and dotted $(z=0)$ lines in Fig. 2c of each $E_{y}$ component, where $X$ is the shifted coordinate crossing the $\mathrm{X}$-point $(x=$ $\left.x_{\text {rec }}\right) . E_{y}$ is almost constant over the reconnection region with $\sim 0.2 V_{\mathrm{Ai}} B_{0}$ as seen in past kinetic studies (e.g., Pritchett, 2001). This finite $E_{y}$ is almost perfectly sustained by $E_{y}^{\mathrm{tot}}=E_{y}^{\mathrm{NG}}+E_{y}^{\mathrm{EI}}+E_{y}^{\mathrm{VB}}$. Note again that since the time $(t=$ $\left.20 \Omega_{i}^{-1}\right)$ shown in Fig. 2 is in a quasi-steady state, $\frac{m_{\mathrm{e}}}{e} \frac{\partial U_{\mathrm{ey}}}{\partial t}$ in $E_{y}^{\mathrm{EI}}$ can be neglected. At the X-point, $E_{y}$ is sustained only by $E_{y}^{\mathrm{NG}}$ as seen in past kinetic studies (e.g., Pritchett, 2001; Ricci et al., 2002; Ishizawa and Horiuchi, 2005; Wang et al., 2015). The positive $E_{y}^{\mathrm{NG}}$ region is surrounded by the finite $E_{y}^{\mathrm{EI}}$ region. With increasing the distance from the X-point, $E_{y}^{\mathrm{VB}}$ increases and reaches $E_{y}$ at the edge of the electron diffusion region (see vertical dashed lines in Fig. 3a and b). Outside the electron diffusion region, $E_{y}^{\mathrm{VB}} \sim E_{y}$ and $E_{y}^{\mathrm{EI}} \sim 0$ and $E_{y}^{\mathrm{NG}} \sim 0$ in the inflow direction (Fig. 3a), while $E_{y}^{\mathrm{VB}}>E_{y}$ and $E_{y}^{\mathrm{EI}}<0$ and $E_{y}^{\mathrm{NG}}<0$ in the outflow direction (Fig. 3b). Past fully kinetic simulations showed that this $E_{y}^{\mathrm{VB}}>E_{y}$ region in the outflow direction extends in larger systems, while the length of the electron diffusion region where $E_{y}^{\mathrm{VB}}<E_{y}$ (i.e., $E_{y}^{\mathrm{rec}}=E_{y}^{\mathrm{EI}}+E_{y}^{\mathrm{NG}}>0$ ) is not significantly affected by the system sizes (e.g., Karimabadi et al., 2007; Shay et al., 2007). Note that although the $E_{y}^{\mathrm{VB}}>E_{y}$ and $E_{y}^{\text {rec }}>0$ regions are sometimes categorized as the outer and inner electron diffusion regions, respectively, in this paper only the $E_{y}^{\text {rec }}>0$ region is defined as the electron diffusion region. In the next three subsections, we will analyze the detailed dimensions of the electron diffusion region where $E_{y}^{\mathrm{rec}}>0$ and the non-gyrotropic region where $E_{y}^{\mathrm{NG}}>0$.

\subsection{Spatial dimension of the electron diffusion region in the $z$ direction}

Figure 4a shows $E_{y}^{\mathrm{rec}}$ and $E_{y}^{\mathrm{NG}}$ along the white dashed line in Fig. $12 \mathrm{c}$ for Run 2. In this paper, we defined the dimensions of the electron diffusion region ( $L_{x}^{\mathrm{Df}}$ and $\left.L_{z}^{\mathrm{Df}}\right)$ and the nongyrotropic region $\left(L_{x}^{\mathrm{NG}}\right.$ and $L_{z}^{\mathrm{NG}}$ ) as the half-lengths between the edges of $E_{y}^{\mathrm{rec}}>0$ and $E_{y}^{\mathrm{NG}}>0$ regions, respectively (see vertical red lines). Note here that only the $|z|=L_{z}^{\text {Df }}$ location is defined as the point where $E_{y}^{\mathrm{rec}}$ falls down to $\left.0.05 E_{y}^{\mathrm{rec}}\right|_{\max }$ since $E_{y}^{\text {rec }}$ decrease asymptotically in the $z$ direction (almost on the $1 / \cosh ^{2}$ curve) with some fluctuations which make it difficult to determine the exact $E_{y}^{\mathrm{rec}}=0$ point. We measured these dimensions for all runs and the results are plotted in Figs. 6 and 9, which shows a clear scaling law for each dimension (less than $3 \%$ of the standard error for each regression line). In this subsection, we discuss the scaling of the dimension of the electron diffusion region in the $z$ direction $\left(L_{z}^{\text {Df }}\right)$. We start here from the $z$ component of the momentum equation of electrons in a steady state at $X=0$,

$\frac{\partial}{\partial z}\left(\frac{1}{2} m_{\mathrm{e}} U_{\mathrm{e} z}(z)^{2}\right) \sim-\frac{1}{n} \frac{\partial P_{\mathrm{e} z}}{\partial z}-e U_{\mathrm{e} y}(z) B_{x}(z)$.

Figure $4 \mathrm{~b}$ shows the profiles in the $z$ direction of each term in Eq. (3). The pressure gradient and the Lorentz force are almost balanced (the inertial term can be negligible) within the electron diffusion region. Assuming that $B_{x}(z)$ varies linearly $\left(B_{x}(z) \sim B^{\prime}{ }_{x} z\right)$, Eq. (3) can roughly be written as

$T_{\mathrm{e} z z}(0)-T_{\mathrm{e} z z}(z) \sim e U_{\mathrm{e} y}(z) \frac{e}{2} B^{\prime}{ }_{x} z^{2}$.

Figure 4d-f show the electron thermal velocity compo-

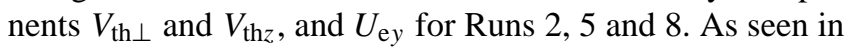



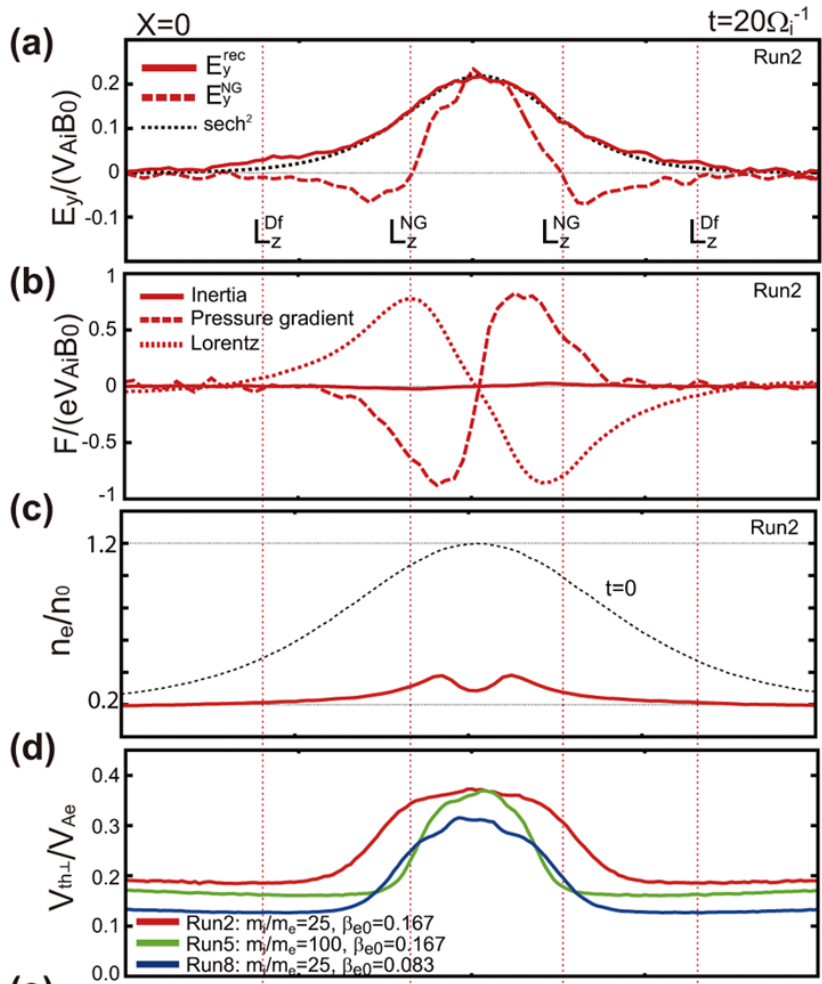

(e)

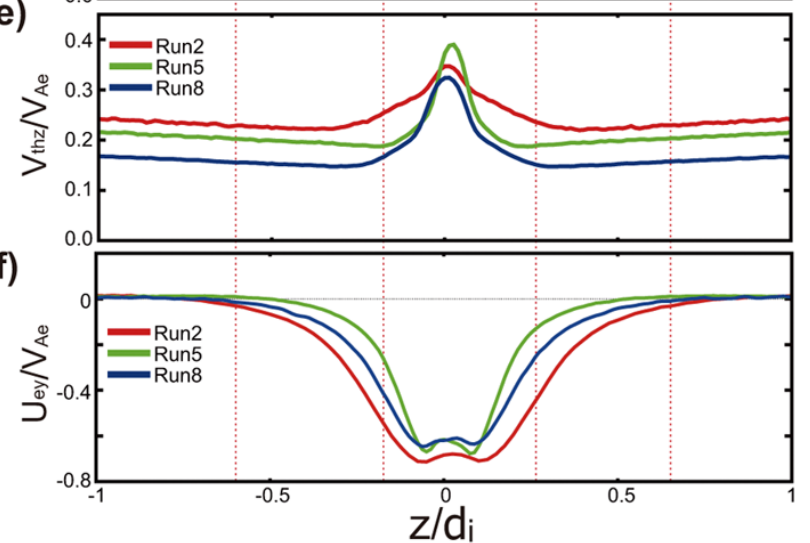

Figure 4. Cuts from the white dashed line in Fig. 2c of $E_{y}^{\text {rec }}$ and $E_{y}^{\mathrm{NG}}(\mathbf{a}), \frac{\partial}{\partial z}\left(\frac{1}{2} m_{\mathrm{e}} U_{\mathrm{e} z}(z)^{2}\right),-\frac{1}{n} \mathrm{e} \frac{\partial P_{\mathrm{e} z}}{\partial z}$ and $-e U_{\mathrm{e} y} B_{x}$ (b) and $n_{\mathrm{e}}$ (c) for Run 2, and $V_{\mathrm{th} \perp}=\sqrt{\left(P_{\mathrm{e} y y}+P_{\mathrm{e} z z}\right) / 2 n}$ (d), $V_{\mathrm{th} z}=$

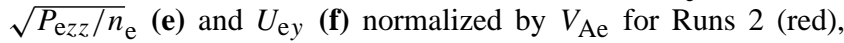
5 (green) and 8 (blue). Vertical lines show the location of $|z|=L_{z}^{\mathrm{Df}}$ and $L_{z}^{\mathrm{NG}}$ for Run 2. The black dotted line in (c) shows the initial density profile.

past kinetic simulations, within the electron diffusion region electrons decoupling from the magnetic field are accelerated by $E_{y}^{\text {rec }}$ (e.g., Pritchett, 2001; Hesse, 2011). This produces the finite $U_{\mathrm{e} y}$ within the diffusion region whose profile is roughly proportional to the electron Alfvén speed $V_{\mathrm{Ae}}$ (see Fig. 4f). In addition, it is known that electrons which cross the current sheet in the positive and negative $z$ directions

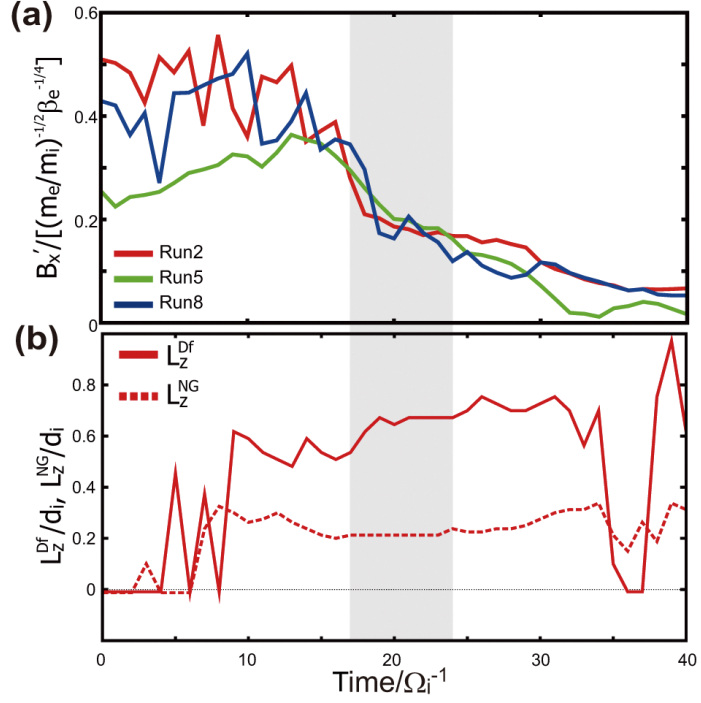

Figure 5. Time evolution of averaged slopes of $B_{x}$ variations in the $z$ direction over $|z|<L_{z}^{\mathrm{Df}}$ divided by $\left(m_{\mathrm{e}} / m_{\mathrm{i}}\right)^{-1 / 2} \beta_{\mathrm{e}}^{-1 / 4}$ for Runs 2 (red), 5 (green) and 8 (blue) (a), and $L_{z}^{\mathrm{Df}}$ and $L_{z}^{\mathrm{NG}}$ for Run 2 (b). The shaded interval indicates the quasi-steady phase for Run 2.

(i.e., meandering electrons) produce a double-peak structure of the perpendicular velocity distributions $F\left(V_{\mathrm{e} y}, V_{\mathrm{e} z}\right)$ at the positive and negative $V_{\mathrm{e} z}$ regions (e.g., $\mathrm{Ng}$ et al., 2012; Bessho et al., 2014; Shuster et al., 2015). Hence, the shape of $F\left(V_{\mathrm{e} y}, V_{\mathrm{e} z}\right)$ and the resulting $T_{\mathrm{e} z z}\left(V_{\mathrm{th} z}\right)$ near the center of the diffusion region is controlled by the speed at which electrons cross the current sheet in the $z$ direction. As seen in Fig. 4e, the increase of $V_{\text {th }}$ near the center $\Delta V_{\text {th } z}$ (the difference between the peak and background values) is roughly proportional to $V_{\mathrm{Ae}}$ even when changing $m_{\mathrm{i}} / m_{\mathrm{e}}$ and $\beta_{\mathrm{e}}$ (i.e., $\left.V_{\text {th0 }}\right)$. From these relations and Eq. (4), we obtained the following relation,

$B^{\prime}{ }_{x} L_{z}^{\mathrm{Df}^{2}} \propto \frac{T_{\mathrm{e} z z}(0)-T_{\mathrm{e} z z}\left(L_{z}^{\mathrm{Df}}\right)}{e V_{\mathrm{Ae}}} \propto \frac{m_{\mathrm{e}}}{e} V_{\mathrm{Ae}}$

As shown in Fig. 5a, the simulation results indicate that $B^{\prime}{ }_{x}$ is roughly proportional to $\left(m_{\mathrm{e}} / m_{\mathrm{i}}\right)^{-1 / 2} \beta_{\mathrm{e}}^{-1 / 4}$. Thus, from Eq. (5), $L_{z}^{\text {Df }}$ would roughly be proportional to $L_{z}^{\mathrm{Df}} \propto$ $\left(m_{\mathrm{e}} / m_{\mathrm{i}}\right)^{1 / 2} \beta_{\mathrm{e}}^{1 / 8} d_{i}=\beta_{\mathrm{e}}^{1 / 8} d_{\mathrm{e}}$.

The solid line in Fig. 5b shows the time evolution of $L_{z}^{\mathrm{Df}}$ for Run 2. $L_{z}^{\mathrm{Df}}$ is roughly constant during the quasi-steady phase (see around $t=20 \Omega_{i}^{-1}$ ). We calculated average values of $L_{z}^{\text {Df }}$ during a quasi-steady phase defined as the time when $\left|\mathrm{d} A_{y} / \mathrm{d} t\right|>0.75\left|\mathrm{~d} A_{y} / \mathrm{d} t\right|_{\max }$ (the shaded interval in Figs. 1 and 5 for Run 2) for Runs 2-8 and plotted the results in Fig. 6a as a function of $\beta_{\mathrm{e}}^{1 / 8} d_{\mathrm{e}}$. The above prediction of the scaling well matches the simulation results especially when taking the background density $n_{\infty}$ for $\beta_{\mathrm{e}}$ and $d_{\mathrm{i}, \mathrm{e}}$. This could be because the density profiles within the diffusion region 
is basically controlled by the density of background plasmas which flow into and fill the diffusion region (see Fig. 4c). The slope of the regression line in Fig. 6a for $n_{\infty}$ is 2.07 with $1.97 \%$ of the standard error. From these results, the dimension of the electron diffusion region in the $z$ direction would roughly be written as

$L_{z}^{\mathrm{Df}} \sim 2 \beta_{\mathrm{e}}^{\frac{1}{8}} d_{\mathrm{e}} \sim 2\left(\frac{m_{\mathrm{e}}}{m_{\mathrm{i}}}\right)^{\frac{1}{2}} \beta_{\mathrm{e}}^{\frac{1}{8}} d_{\mathrm{i}}$,

where $n=n_{\infty}, B=B_{0}$, and $T_{\mathrm{i}, \mathrm{e}}=T_{\mathrm{i} 0, \mathrm{e} 0}$ are used to obtain $\beta_{\mathrm{e}}$ and $d_{\mathrm{i}, \mathrm{e}}$. This scaling is consistent with the past kinetic simulations which suggested the thickness of the electron diffusion region is determined by the electron inertial length $d_{\mathrm{e}}$ when $\beta_{\mathrm{e}}$ is fixed (e.g., Shay et al., 2001; Zeiler et al., 2002; Dorfman et al., 2008).

In addition, when $\Delta V_{\text {th }}\left(\propto V_{\mathrm{Ae}}\right)$ is sufficiently larger than $V_{\text {th0 }}$ (i.e., $\beta_{\mathrm{e}}$ is sufficiently low), $V_{\text {thz }}$ near the $\mathrm{X}$-point would roughly be proportional to $V_{\mathrm{Ae}}$, as seen in Fig. 4e. In such a low $\beta_{\mathrm{e}}$ situation, from Eq. (5), $B^{\prime}{ }_{x} L_{z} \mathrm{Df}^{2} \propto m_{\mathrm{e}} V_{\text {thz }} / e$. Since the flux $U_{\mathrm{e} z} B_{x}$ reaches $E_{y}$ near $|z|=L_{z}^{\mathrm{Df}}$, assuming the linearity, $U^{\prime}{ }_{\mathrm{e} z} B^{\prime}{ }_{x} L_{z} \mathrm{Df}^{2} \sim E_{y}$. Thus, $E_{y}$ can roughly be proportional to $E_{y} \propto \frac{m_{\mathrm{e}}}{e} V_{\mathrm{th} z} U_{\mathrm{e} z}^{\prime} \sim \frac{\sqrt{m_{\mathrm{e}} T_{\mathrm{ezz}}}}{e} U_{\mathrm{e} z}^{\prime}$. This expression is consistent with past theories of $E_{y}$ component produced by the non-gyrotropic effects near the X-point $\left(E_{y}^{\mathrm{NG}}\right)$ (e.g., Kuznetsova et al., 1998; Hesse et al., 1999; Dorfman et al., 2008; Divin et al., 2012), in which they derived

$\left.E_{y}^{\mathrm{NG}}\right|_{\text {theory }} \sim \frac{\sqrt{2 m_{\mathrm{e}} T_{\mathrm{e} z z}}}{e} U_{\mathrm{e} z}^{\prime} \sim \frac{\sqrt{2 m_{\mathrm{e}} T_{\mathrm{e} x x}}}{e} U_{\mathrm{e} x}^{\prime}$.

Dashed and dotted lines in Fig. 1 show $E_{y}^{\mathrm{NG}}, \frac{\sqrt{2 m_{\mathrm{e}} T_{\mathrm{e} x x}}}{e} U_{\mathrm{e} x}^{\prime}$ and $\frac{\sqrt{2 m_{\mathrm{e}} T_{\mathrm{e} z}}}{e} U_{\mathrm{e} z}^{\prime}$ at the X-point, respectively. The result indeed shows that Eq. (7) is almost true (that is, $E_{y}^{\mathrm{NG}} \sim$ $\left.\left.E_{y}^{\mathrm{NG}}\right|_{\text {theory }}\right)$.

\subsection{Spatial dimension of the non-gyrotropic region in the $z$ direction}

The dimension of the non-gyrotropic region in the $z$ direction $L_{z}^{\mathrm{NG}}$ is believed to be determined by the width of the meandering motion near the X-point (e.g., Kuznetsova et al., 1998; Hesse et al., 1999). The meandering width is known to be described by the $z$ coordinate where the local gyroradius of electrons exceeds the $z$ coordinate (that is, $\rho_{e}(z)>z$ ) (e.g., Biskamp and Schindler, 1971; Horiuchi and Sato, 1994; Kuznetsova et al., 1998). Assuming that $B_{x}(z)$ varies linearly and the perpendicular thermal velocity $V_{\text {th } \perp}$ is constant near the meandering region, the $z$ coordinate at the edge of the meandering region $\left(z=L_{z}^{\mathrm{NG}}\right)$ can be described as $\rho_{\mathrm{e}}(z) \sim \rho_{\mathrm{e}} L_{z}^{B} / z \sim z$, where $\rho_{\mathrm{e}}=m_{\mathrm{e}} V_{\mathrm{th} \perp} /\left(e B_{0}\right)$ and $L_{z}^{B}=$ $B_{0} / B^{\prime}{ }_{x}$ is the magnetic field gradient scale. Thus, the meandering width that corresponds to the dimension of the nongyrotropic region can be described as $L_{z}^{\mathrm{NG}} \sim\left(\rho_{\mathrm{e}} L_{z}^{B}\right)^{1 / 2}$. As
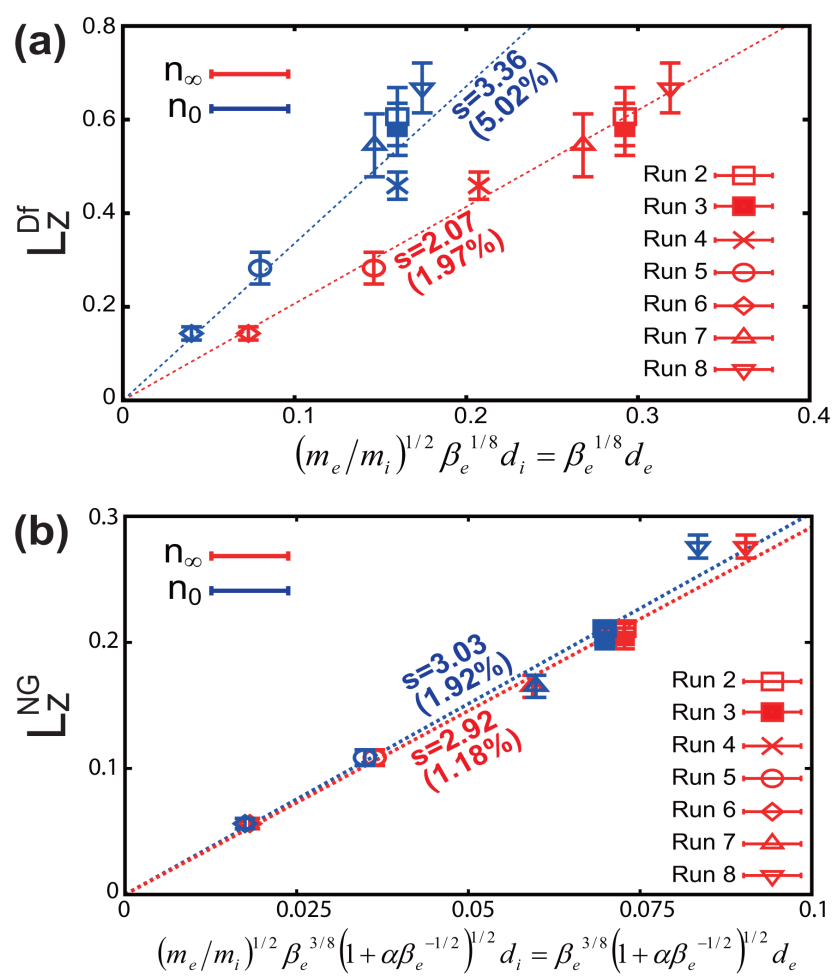

Figure 6. $L_{z}^{\mathrm{Df}}$ versus $\left(m_{\mathrm{e}} / m_{\mathrm{i}}\right)^{1 / 2} \beta_{\mathrm{e}}^{1 / 8} d_{\mathrm{i}}=\beta_{\mathrm{e}}^{1 / 8} d_{\mathrm{e}} \quad$ (a), and $L_{z}^{\mathrm{NG}}$ versus $\left(m_{\mathrm{e}} / m_{\mathrm{i}}\right)^{1 / 2} \beta_{\mathrm{e}}^{3 / 8} \sqrt{1+\alpha \beta_{\mathrm{e}}^{-\frac{1}{2}}} d_{\mathrm{i}}=\beta_{\mathrm{e}}^{3 / 8} \sqrt{1+\alpha \beta_{\mathrm{e}}^{-\frac{1}{2}}} d_{\mathrm{e}}$ where $\alpha=0.15$ (b) normalized by $d_{\mathrm{i} 0}$ for Runs 2-8. $n_{\infty}$ (red), and $n_{0}$ (blue) are examined to calculate $d_{\mathrm{e}}$ and $d_{\mathrm{i}}$. Each value is the averaged one during a quasi-steady period where $\left|\mathrm{d} A_{y} / \mathrm{d} t\right|>$ $0.75\left|\mathrm{~d} A_{y} / \mathrm{d} t\right|_{\max }$. Error bars show the standard deviation, and the dashed lines indicates the regression lines with slopes and standard errors.

seen in Fig. 4d, $V_{\text {th } \perp}$ is enhanced within the diffusion region and roughly constant within the meandering region. The increase of $V_{\text {th }}$ from the background region is roughly proportional to $V_{\mathrm{Ae}}$ (i.e., $V_{\mathrm{th} \perp} \sim V_{\mathrm{th} 0}+\alpha V_{\mathrm{Ae}}$ ) even when changing $m_{\mathrm{i}} / m_{\mathrm{e}}$ and $\beta_{\mathrm{e}}$. From Fig. $4 \mathrm{~d}$, the acceleration rate $\alpha$ is roughly 0.15 for all runs.

Since $L_{z}^{B} \propto\left(m_{\mathrm{e}} / m_{\mathrm{i}}\right)^{1 / 2} \beta_{\mathrm{e}}^{1 / 4}$ as shown in Fig. 5a, $L_{z}^{\mathrm{NG}}$ would be proportional to

$L_{z}^{\mathrm{NG}} \propto\left(\frac{m_{\mathrm{e}}}{m_{\mathrm{i}}}\right)^{\frac{1}{2}} \beta_{\mathrm{e}}^{\frac{3}{8}} \sqrt{1+\alpha \beta_{\mathrm{e}}^{-\frac{1}{2}}} d_{\mathrm{i}}=\beta_{\mathrm{e}}^{\frac{3}{8}} \sqrt{1+\alpha \beta_{\mathrm{e}}^{-\frac{1}{2}}} d_{\mathrm{e}}$.

As shown in Fig. $6 \mathrm{~b}$ which shows the averaged $L_{z}^{\mathrm{NG}}$ during a quasi-steady phase for Runs $2-8$, this prediction of the scaling well matches the simulation results especially when taking the background density $n_{\infty}$ for $\beta_{\mathrm{e}}$ and $d_{\mathrm{i}, \mathrm{e}}$. The slope of the regression lines in Fig. $6 \mathrm{~b}$ for $n_{\infty}$ is 2.92 with $1.18 \%$ of the standard error. From these results, the dimension of the electron diffusion region in the $z$ direction would roughly be 

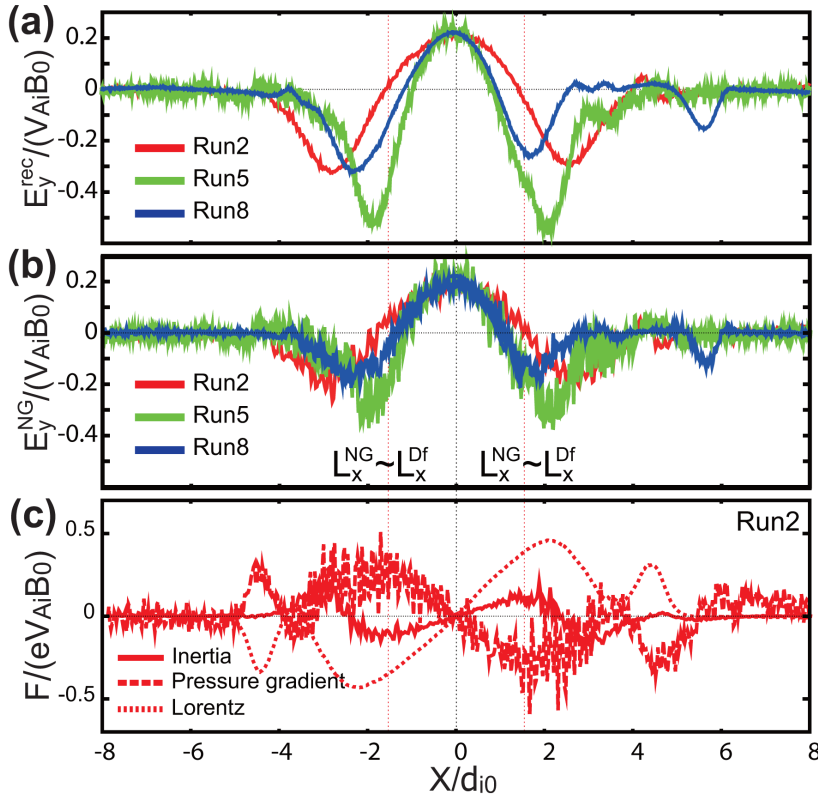

Figure 7. Cuts from the white dotted line in Fig. 2c of $E_{y}^{\mathrm{rec}}$ (a) and $E_{y}^{\mathrm{NG}}$ (b) for Runs 2 (red), 5 (green) and 8 (blue), and $m_{\mathrm{e}} U_{\mathrm{e} x} \frac{\partial U_{\mathrm{e} x}}{\partial x}$, $-\frac{1}{n} \mathrm{e} \frac{\partial P_{\mathrm{e} x x}}{\partial x}$ and $e U_{\mathrm{e} y} B_{z}$ for Run 2 (c). Vertical lines in Fig. 7a-c show the location of $|X|=L_{x}^{\mathrm{Df}} \sim L_{x}^{\mathrm{NG}}$ for Run 2 .

written as

$$
\begin{aligned}
L_{z}^{\mathrm{NG}} & \sim 3 \beta_{\mathrm{e}}^{\frac{3}{8}} \sqrt{1+\alpha \beta_{\mathrm{e}}^{-\frac{1}{2}}} d_{\mathrm{e}} \\
& \sim 3\left(\frac{m_{\mathrm{e}}}{m_{\mathrm{i}}}\right)^{\frac{1}{2}} \beta_{\mathrm{e}}^{\frac{3}{8}} \sqrt{1+\alpha \beta_{\mathrm{e}}^{-\frac{1}{2}}} d_{\mathrm{i}},
\end{aligned}
$$

where $n=n_{\infty}, B=B_{0}$, and $T_{\mathrm{i}, \mathrm{e}}=T_{\mathrm{i} 0, \mathrm{e} 0}$ are used to obtain $\beta_{\mathrm{e}}$ and $d_{\mathrm{i}, \mathrm{e}}$.

\subsection{Spatial dimensions in the $x$ direction}

As seen in Fig. 3b, $E_{y}^{\mathrm{EI}}$ is basically negative on the $x$ axis, and the negative $E_{y}^{\mathrm{EI}}$ peaks are seen outside the positive $E_{y}^{\mathrm{NG}}$ region. Hence, the positive $E_{y}^{\text {rec }}$ region in the $x$ direction almost corresponds to the positive $E_{y}^{\mathrm{NG}}$ region (i.e., $L_{x}^{\mathrm{Df}} \sim L_{x}^{\mathrm{NG}}$ ) as seen in Fig. 7a and $b$ (see vertical lines for Run 2). In this subsection, we discuss the dimension $L_{x}^{\mathrm{Df}}\left(\sim L_{x}^{\mathrm{NG}}\right)$ of the $E_{y}^{\mathrm{rec}}>0$ region in the $x$ direction. Figure $7 \mathrm{c}$ shows the profiles along the $x$ axis (i.e., $z=0$ ) of each term of the $x$ component of the momentum equation, $m_{\mathrm{e}} U_{\mathrm{e} x} \frac{\partial U_{\mathrm{e} x}}{\partial x},-\frac{1}{n} \mathrm{e} \frac{\partial P_{\mathrm{e} x x}}{\partial x}$ and $e U_{\mathrm{e} y} B_{z}$ for Run 2 . In contrast to the profiles in the $z$ direction, the inertial term cannot be negligible and therefore the same approach to derive the scaling as shown in Sect. 3.2 cannot be applied to $L_{x}^{\mathrm{Df}}$. Instead, we start here from the approximated $E_{y}^{\mathrm{NG}}$ described in Eq. (7), which is almost equal to the constant $E_{y}$ value within the diffusion region as shown in Sect. 3.2. Assuming the linearity in the $x$ direction and the flux conservation at the edge of the electron diffusion region
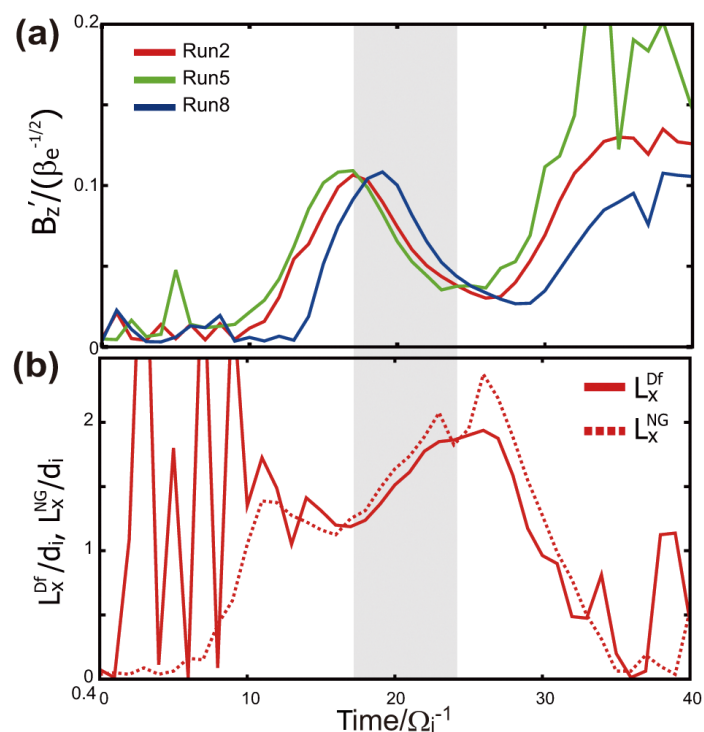

Figure 8. Time evolution of averaged slopes of $B_{z}$ variations in the $z$ direction over $|X|<L_{x}^{\mathrm{Df}}$ divided by $\beta_{\mathrm{e}}^{-1 / 2}$ for Runs 2 (red), 5 (green) and 8 (blue) (a), and $L_{x}^{\mathrm{Df}}$ and $L_{x}^{\mathrm{NG}}$ for Run 2 (b). The shaded interval indicates the quasi-steady phase for Run 2.

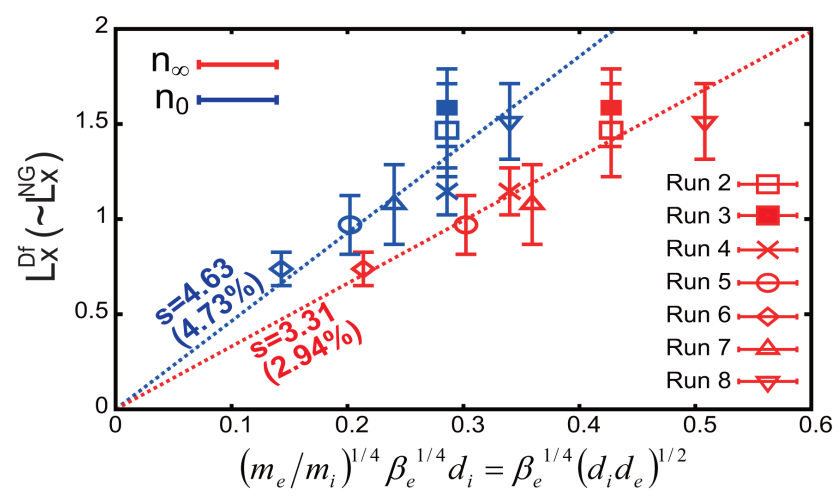

Figure 9. $L_{x}^{\mathrm{Df}}\left(\sim L_{x}^{\mathrm{NG}}\right)$ versus $\left(m_{\mathrm{e}} / m_{\mathrm{i}}\right)^{1 / 4} \beta_{\mathrm{e}}^{1 / 4} d_{\mathrm{i}}$ normalized by $d_{\mathrm{i} 0}$ for Runs $2-8$. $n_{\infty}$ (red), and $n_{0}$ (blue) are examined to calculate $d_{\mathrm{e}}$ and $d_{\mathrm{i}}$. Each value is the averaged one during a quasi-steady period. Error bars show the standard deviation, and the dashed lines indicates the regression lines with slopes and standard errors.

(i.e., $U^{\prime}{ }_{\text {ex }} B^{\prime}{ }_{z} L_{x}^{\mathrm{Df}^{2}} \sim E_{y}$ ), from Eq. (7), $L_{x}^{\mathrm{Df}}$ can roughly be written as

$L_{x}^{\mathrm{Df}} \sim\left[\frac{1}{B^{\prime}{ }_{z}} \frac{\sqrt{2 m_{\mathrm{e}} T_{\mathrm{e} x x}}}{e}\right]^{\frac{1}{2}} \sim\left(\frac{m_{\mathrm{e}}}{m_{\mathrm{i}}}\right)^{\frac{1}{4}}\left(\frac{B_{0}}{B^{\prime}{ }_{z} d_{\mathrm{i}}}\right)^{\frac{1}{2}} \beta_{\mathrm{e}}^{\frac{1}{2}} d_{\mathrm{i}}$.

As shown in Fig. 8a, the simulation results indicate that $B_{z}^{\prime}$ is roughly proportional to $\beta_{\mathrm{e}}^{-1 / 2}$. Thus, from Eq. (10), $L_{x}^{\mathrm{Df}}\left(\sim L_{x}^{\mathrm{NG}}\right)$ would roughly be proportional to $\left(m_{\mathrm{e}} / m_{\mathrm{i}}\right)^{1 / 4} \beta_{\mathrm{e}}^{1 / 4} d_{\mathrm{i}}=\beta_{\mathrm{e}}^{1 / 4}\left(d_{\mathrm{i}} d_{\mathrm{e}}\right)^{1 / 2}$. As shown in Fig. 9 which shows the averaged $L_{x}^{\mathrm{Df}}$ during a quasi-steady phase for Runs 2-8, this prediction of the scaling well matches the sim- 
ulation results especially when taking the background density $n_{\infty}$ for $\beta_{\mathrm{e}}$ and $d_{\mathrm{i}, \mathrm{e}}$. The slope of the regression line in Fig. 9 for $n_{\infty}$ is 3.31 with $2.94 \%$ of the standard error. From these results, the dimension of the electron diffusion region in the $x$ direction would roughly be written as

$L_{x}^{\mathrm{Df}} \sim L_{x}^{\mathrm{NG}} \sim 3 \beta_{\mathrm{e}}^{\frac{1}{4}}\left(d_{\mathrm{i}} d_{\mathrm{e}}\right)^{\frac{1}{2}}$,

where $n=n_{\infty}, B=B_{0}$, and $T_{\mathrm{i}, \mathrm{e}}=T_{\mathrm{i} 0, \mathrm{e} 0}$ are used to obtain $\beta_{\mathrm{e}}$ and $d_{\mathrm{i}, \mathrm{e}}$. Similar scalings were also seen in past kinetic simulations (Hesse et al., 1999; Ricci et al., 2002; Daughton et al., 2006; Karimabadi et al., 2007; Divin et al., 2012), where they indicated that the dimension of the electron diffusion region in the $x$ direction is proportional to $\left(m_{\mathrm{e}} / m_{\mathrm{i}}\right)^{1 / 4}$ when $\beta_{\mathrm{e}}$ is fixed.

The same scaling of $L_{x}^{\mathrm{Df}}$ can also be derived from the flux conservation at both edges of the electron diffusion region in the inflow and outflow directions $U_{\text {ein }} B_{\text {in }} \sim U_{\text {eout }} B_{\text {out }}$ where $U_{\text {ein }}=U_{\mathrm{ez}}\left(L_{z}^{\mathrm{Df}}\right), U_{\text {eout }}=U_{\mathrm{e} x}\left(L_{x}^{\mathrm{Df}}\right), B_{\mathrm{in}}=B_{z}\left(L_{z}^{\mathrm{Df}}\right)$, and $B_{\text {out }}=B_{z}\left(L_{x}^{\mathrm{Df}}\right)$. Assuming the linearity and the incompressibility (which lead to $U_{\text {ein }} / L_{z}^{\mathrm{Df}} \sim U_{\text {eout }} / L_{x}^{\mathrm{Df}}$ ), the above flux conservation can be rewritten as $B_{\text {in }} / B_{\text {out }} \sim$ $L_{x}^{\mathrm{Df}} / L_{z}^{\mathrm{Df}}$. From this relation and the scalings used in Sect. 3.2 and this section $\left(B^{\prime}{ }_{x}\left(\sim B_{\text {in }} / L_{z}^{\mathrm{Df}}\right), B^{\prime}{ }_{z}\left(\sim B_{\text {out }} / L_{x}^{\mathrm{Df}}\right)\right.$ and $\left.L_{z}^{\mathrm{Df}}\right)$, we can obtain the relation shown in Eq. (11) $L_{x}^{\mathrm{Df}} \propto$ $\left(m_{\mathrm{e}} / m_{\mathrm{i}}\right)^{1 / 4} \beta_{\mathrm{e}}^{1 / 4} d_{\mathrm{i}}$. This is not surprising since the theoretical $E_{y}$ at the X-point (Eq. 7) is basically the same value as the flux $\left(U_{\text {ein }} B_{\text {in }}\right)$ at the edge of the electron diffusion region as shown in Sect. 3.2.

\section{Summary and discussions}

In this paper, based on 2-D fully kinetic PIC simulations we systematically analyzed the dimensions of the whole electron diffusion region of anti-parallel reconnection ( $L_{x}^{\mathrm{Df}}$ and $L_{z}^{\mathrm{Df}}$ ) and the non-gyrotropy dominant region located near the center of the diffusion region $\left(L_{x}^{\mathrm{NG}}\right.$ and $\left.L_{z}^{\mathrm{NG}}\right)$. The simulations with sufficiently small grid spacing and large number of particles allowed us to successfully obtain the following precise scalings of the dimensions with sufficiently small errors (see regression lines and bars in Figs. 6 and 9),

$$
\begin{aligned}
& L_{z}^{\mathrm{Df}} \sim 2 \beta_{\mathrm{e}}^{\frac{1}{8}} d_{\mathrm{e}} \sim 25 \times\left(\frac{\beta_{\mathrm{e}}}{0.1}\right)^{\frac{1}{8}}\left(\frac{0.1 \mathrm{~cm}^{-3}}{n}\right)^{\frac{1}{2}} \mathrm{~km}, \\
& L_{z}^{\mathrm{NG}} \sim 3 \beta_{\mathrm{e}}^{\frac{3}{8}} \sqrt{1+\alpha \beta_{\mathrm{e}}^{-\frac{1}{2}}} d_{\mathrm{e}} \sim \\
& 25 \times\left(\frac{\beta_{\mathrm{e}}}{0.1}\right)^{\frac{3}{8}}\left(1+0.15\left(\frac{0.1}{\beta_{\mathrm{e}}}\right)^{\frac{1}{2}}\right)^{\frac{1}{2}}\left(\frac{0.1 \mathrm{~cm}^{-3}}{n}\right)^{\frac{1}{2}} \mathrm{~km},
\end{aligned}
$$

$$
\begin{aligned}
& L_{x}^{\mathrm{Df}} \sim L_{x}^{\mathrm{NG}} \sim 3 \beta_{\mathrm{e}}^{\frac{1}{4}}\left(d_{\mathrm{i}} d_{\mathrm{e}}\right)^{\frac{1}{2}} \sim \\
& 200 \times\left(\frac{\beta_{\mathrm{e}}}{0.1}\right)^{\frac{1}{4}}\left(\frac{0.1 \mathrm{~cm}^{-3}}{n}\right)^{\frac{1}{2}} \mathrm{~km},
\end{aligned}
$$

where we take $m_{\mathrm{i}} / m_{\mathrm{e}}=1836$ to estimate the real values. The density and $\beta_{\mathrm{e}}$ for all dimensions are taken as the values in the background region. The results show that the dimensions in the inflow direction are basically proportional to the electron inertial length, while those in the outflow direction are proportional to the ion-electron hybrid inertial length. The dependence on $\beta_{\mathrm{e}}$ (the electron temperature) is different for each dimension. As shown in Sects. 3.2-3.4, these scalings are in good agreement with theories that we extended to predict the scalings by considering the spatial variations of field and plasma parameters within the diffusion region. In the theories, the above scaling laws can be predicted by inputting the gradients of the magnetic field in the inflow $\left(B^{\prime}{ }_{x}\right)$ and outflow $\left(B^{\prime}{ }_{z}\right)$ directions within the diffusion region which are obtained from the simulations - i.e., the scalings are determined self-consistently when the magnetic field gradients are given. Shay et al. (2007) showed that even when considering larger system sizes both the reconnection rate and the gradient in the outflow direction $B^{\prime}{ }_{z}$ near the X-point are not significantly changed. This could be the reason why the length of the electron diffusion region in the outflow direction where $E_{y}^{\mathrm{VB}}<E_{y}$ ( $L_{x}^{\mathrm{Df}}$ in this paper) is not significantly affected by the system sizes, despite the outer region where $E_{y}^{\mathrm{VB}}>E_{y}$ can be extended depending on the system sizes (e.g., Karimabadi et al., 2007; Shay et al., 2007).

The scalings obtained in this paper lead to important implications for spacecraft observations in the Earth's magnetotail. A recent statistical study using the Cluster spacecraft showed that the reconnection X-line in the magnetotail tends to move tailward ( $\sim x$ direction in this paper) at about $70 \mathrm{~km} \mathrm{~s}^{-1}$ on average (Alexandrova et al., 2015). This tendency is consistent with past event studies (e.g., Baker et al., 2002; Nagai et al., 2011), in which the tailward X-line speed was estimated as the order of $100 \mathrm{~km} \mathrm{~s}^{-1}$. Assuming that the X-line moves in the $x$ direction at $U_{\mathrm{r} x}=50-100 \mathrm{~km} \mathrm{~s}^{-1}$ and the spacecraft is fixed near the center of the plasma sheet $(z \sim 0)$, from Eq. (14), the observation duration for the electron diffusion region when $0.01<n<0.1$ can roughly be predicted as $\Delta t_{x}=2 L_{x}^{\mathrm{Df}} / U_{\mathrm{r} x} \sim 5-30 \mathrm{~s}$ when $\beta_{\mathrm{e}} \sim 0.1$. Here $n$ roughly corresponds to the local density near the diffusion region as shown in Sect. 3.2. This prediction is roughly consistent with recent observational studies using the Geotail spacecraft (Nagai et al., 2011, 2013), in which they showed direct observations of the vicinity of the X-line where ion and electron reconnection outflows are separated. In their observations, the peak-to-peak duration of electron outflows were about 20-30 s for some representative events and less than 1-2 min. on average. The density in these intervals was roughly comparable or less than $0.1 \mathrm{~cm}^{-3}$. Since 
the peak-to-peak distance of the electron outflows is roughly two times larger than $2 L_{x}^{\mathrm{Df}}$ (see the peak-to-peak distance of blue line in Fig. 3b), the expected peak-to-peak duration when $0.01<n_{\infty}<0.1$ is roughly $10 \mathrm{~s}<\Delta t<1 \mathrm{~min}$, which is roughly consistent with the observations.

Although the above observation duration of the electron diffusion region predicted from our simulations $\left(\Delta t_{x}=5\right.$ $30 \mathrm{~s})$ is calculated under the assumption that the spacecraft is fixed near the center of the plasma sheet (i.e., the X-line motion speed in the $z$ direction $U_{\mathrm{r} z}$ is small enough), the observation duration would also be sensitive to $U_{\mathrm{r} z}$. We here introduce $\tau$ which is defined as the ratio of the observation durations between the $z$ and $x$ directions,

$$
\begin{aligned}
\tau & =\frac{\Delta t_{z}}{\Delta t_{x}}=\frac{2 L_{z}^{\mathrm{Df}} / U_{\mathrm{r} z}}{2 L_{x}^{\mathrm{Df}} / U_{\mathrm{r} x}} \\
& =\frac{L_{z}^{\mathrm{Df}}}{L_{x}^{\mathrm{Df}}} \frac{U_{\mathrm{r} x}}{U_{\mathrm{r} z}} \sim \frac{2}{3} \beta_{\mathrm{e}}^{-\frac{1}{8}}\left(\frac{m_{\mathrm{e}}}{m_{\mathrm{i}}}\right)^{\frac{1}{4}} \frac{U_{\mathrm{r} x}}{U_{\mathrm{r} z}} \sim 0.13 \times\left(\frac{\beta_{\mathrm{e}}}{0.1}\right)^{-\frac{1}{8}} \frac{U_{\mathrm{r} x}}{U_{\mathrm{r} z}} .
\end{aligned}
$$

When $\tau>1$ the observed data reflect the change of the structure of the electron diffusion region in the $x$ direction rather than that in the $z$ direction, and vice versa. From Eq. (15), the threshold $\tau=1$ corresponds to $U_{\mathrm{r} z} / U_{\mathrm{r} x} \sim 0.1$ when $\beta_{\mathrm{e}} \sim$ 0.1 . Although it is difficult to know $U_{\mathrm{r} z} / U_{\mathrm{r} x}$ in the above Geotail events, the consistency with the predicted duration in the $\mathrm{x}$ direction implies $\tau>1$ (i.e., $U_{\mathrm{r} z} / U_{\mathrm{r} x}<0.1$ ) in these events.

On the other hand, to analyze the structure of the electron diffusion region in the $z$ direction, the condition with $\tau<1$ (i.e., $U_{\mathrm{r} z} / U_{\mathrm{r} x}>0.1$ ) would be required. The statistical study of the X-line motion using Cluster (Alexandrova et al., 2015), which analyzed reconnection events following current sheet crossings, showed that the $\mathrm{X}$-line tends to move in the north-south $(\sim z)$ direction correlated with the motion of the current sheet, and its motion speed is about $30 \mathrm{~km} \mathrm{~s}^{-1}$ on average and varies up to $100-200 \mathrm{~km} \mathrm{~s}^{-1}$ (corresponding to $\Delta t_{z}=0.2-5 \mathrm{~s}$ when $0.01<n_{\infty}<0.1$ and $\left.\beta_{\mathrm{e}} \sim 0.1\right)$. Since the averaged speed in the $x$ direction in Alexandrova et al. (2015) is $70 \mathrm{~km} \mathrm{~s}^{-1}$, these $X$-line motion speeds indicate that the case with $\tau<1\left(U_{\mathrm{r} z} / U_{\mathrm{r} x}>0.1\right)$ would not be uncommon at least for reconnection events with current sheet crossings. Since the expected observation duration of the electron diffusion region for such events $(0.2-5 \mathrm{~s})$ is comparable to or less than typical spin periods (i.e., the time resolution) of spacecraft such as Geotail, Cluster or THEMIS, the detailed structure of the electron diffusion region is expected to be realized for the first time by further comparison with the MMS mission which measures particles with millisecond resolution.

Although this paper treated an anti-parallel magnetic field configuration, effects of the guide magnetic field component, which often exists for the dayside reconnection (e.g., Swisdak et al., 2003; Hesse, 2006) or the vortex-induced reconnection (e.g., Nakamura et al., 2013) at the Earth's magnetopause, should be examined to more generally understand the structure of the electron diffusion region. Since electrons are more strongly magnetized even near the X-point when considering the guide field, the orbit of electrons near the $\mathrm{X}$ point and the associated structure of the non-gyrotropic region would significantly be affected by the guide field as indicated in past kinetic studies (e.g., Horiuchi and Sato, 1997; Ricci et al., 2004; Swisdak et al., 2005; Hesse, 2006). In addition, the strongly magnetized electrons in the guide field case would easily be trapped around the ion and electron diffusion regions (e.g., Egedal et al., 2008), and these trapped electrons would affect the structures and the dimensions of the diffusion regions. Considering such guide field effects would be an important future research topic.

Non-steady features, which are also neglected in this paper, should also be considered to understand the electronscale physics of magnetic reconnection. Recent 2-D and 3-D kinetic simulations demonstrated that repeated formation of secondary small-scale flux ropes near the X-line and within the reconnection exhausts drastically disturb steady reconnection features (e.g., Daughton et al., 2006; Fujimoto and Sydora, 2012; Lapenta et al., 2015). Furthermore, recent 3-D fully kinetic simulations also demonstrated that when considering the strong guide field component, similar turbulent features easily spread even outside these regions and fill the whole reconnection layer through the copious formation of oblique secondary flux ropes (Daughton et al., 2011, 2014). Spacecraft observations using Cluster indeed indicated the existence of turbulence near the X-line in the magnetotail (Eastwood et al., 2009). Understanding the structure of the diffusion region in such non-steady situations would also be an important future research topic.

Acknowledgements. This work is supported by Austrian Science Fund (FWF): P23862-N16, I2016-N20. Simulations were performed with resources from the LANL institutional computing program. The large-scale simulation data in this study are available upon request from the authors. We would like to acknowledge useful discussions with W. Baumjohann, W. Daughton, I. Shinohara and S. Zenitani.

The topical editor, C. Owen, thanks two anonymous referees for help in evaluating this paper.

\section{References}

Alexandrova, A., Nakamura, R., Semenov, V. S., and Nakamura, T. K. M.: Motion of reconnection region in the Earth's magnetotail, Geophys. Res. Lett., 42, 4685-4693, doi:10.1002/2015GL064421, 2015.

Baker, D. N., Peterson, W. K., Eriksson, S., Li, X., Blake, J. B., Burch, J. L., Daly, P. W., Dunlop, M. W., Korth, A., Donovan, E., Friedel, R., Fritz, T. A., Frey, H. U., Mende, S. B., Roeder, J., and Singer, H. J.: Timing of magnetic reconnection initiation during a global magnetospheric substorm onset, Geophys. Res. Lett., 29, 2190, doi:10.1029/2002GL015539, 2002.

Bessho, N., Chen, L.-J., Shuster, J. R., and Wang, S.: Electron distribution functions in the electron diffusion region of magnetic 
reconnectoon: Physics behond the fine structures, Geophys. Res. Lett., 41, 8688, doi:10.1002/2014GL062034, 2014.

Birn, J., Drake, J. F., Shay, M. A., Rogers, B. N., Denton, R. E., Hesse, M., Kuznetsova, M., Ma, Z. W., Bhattacharjee, A., Otto, A., and Pritchett, P. L.: Geospace Environmental Modeling (GEM) Magnetic Reconnection Challenge, J. Geophys. Res., 106, 3715-3719, doi:10.1029/1999JA900449, 2001.

Birn, J. and Priest, E. R.: Reconnection of Magnetic Fields : Magnetohydrodynamics and Collisionless Theory and Observations, Cambridge University Press, Cambridge, UK, 2007.

Biskamp, D. and Schindler, K.: Instability of two-dimensional collisionless plasmas with neutral points, Plasma Phys., 13, 1013, doi:10.1088/0032-1028/13/11/003, 1971.

Bowers, K. J., Albright, B. J., Yin, L., Bergen, B., and Kwan, T. J. T.: Ultrahigh performance three-dimensional electromagnetic relativistic kinetic plasma simulation, Phys. Plasmas, 15, 055703, doi:10.1063/1.2840133, 2008.

Daughton, W., Scudder, J., and Karimabadi, H.: Fully kinetic simulations of undriven magnetic reconnection with open boundary conditions, Phys. Plasmas, 13, 072101, doi:10.1063/1.2218817, 2006.

Daughton, W., Roytershteyn, V., Karimabadi, H., Yin, L., Albright, B. J., Bergen, B., and Bowers, K. J.: Role of electron physics in the development of turbulent magnetic reconnection in collisionless plasmas, Nature Phys., 7, 539-542, doi:10.1038/nphys1965, 2011.

Daughton, W., Nakamura, T. K. M., Karimabadi, H., Roytershteyn, V., and Loring, B.: Computing the reconnection rate in turbulent kinetic layers by using electron mixing to identify topology, Phys. Plasmas, 21, 252307, doi:10.1063/1.4875730, 2014.

Divin, A., Lapenta, G., Markidis, S., Semenov, V. S., Erkaev, N. V., Korovinskiy, D. B., and Biernat, H. K.: Scaling of the inner electron diffusion region in collisionless magnetic reconnection, J. Geophys. Res., 117, A06217, doi:10.1029/2011JA017464, 2012.

Dorfman, S., Daughton, W., Roytershteyn, V., Ji, H., Ren, Y., and Yamada, M.: Two-dimensional fully kinetic simulations of driven magnetic reconnection with boundary conditions relevant to the Magnetic Reconnection Experiment, Phys. Plasmas, 15, 102107, doi:10.1063/1.2991361, 2008.

Eastwood, J. P., Phan, T. D., Bale, S. D., and Tjulin, A.: Observations of Turbulence Generated by Magnetic Reconnection, Phys. Rev. Lett., 102, 035001, doi:10.1103/PhysRevLett.102.035001, 2009.

Egedal, J., Fox, W., Katz, N., Porkolab, M., Øieroset, M., Lin, R. P., Daughton, W., and Drake, J. F.: Evidence and theory for trapped electrons in guide field magnetotail reconnection, J. Geophys. Res., 113, A12207, doi:10.1029/2008JA013520, 2008.

Fujimoto, K. and Sydora, R. D.: Plasmoid-Induced Turbulence in Collisionless Magnetic Reconnection, Phys. Rev. Lett., 109, 265004, doi:10.1103/PhysRevLett.109.265004, 2012.

Hesse, M. and Winske, D.: Electron dissipation in collisionless magnetic reconnection, J. Geophys. Res., 103, 26479-26486, 1998.

Hesse, M., Schindler, K., Birn, J., and Kuznetsova, M.: The diffusion region in collisionless magnetic reconnection, Phys. Plasmas, 6, 1781, doi:10.1063/1.873436, 1999.

Hesse, M.: Dissipation in magnetic reconnection with a guide magnetic field, Phys. Plasmas, 13, 122107, doi:10.1063/1.2403784, 2006.
Hesse, M., Neukirch, T., Schindler, K., Kuznetsova, M., and Zenitani, S.: The Diffusion Region in Collisionless Magnetic Reconnection, Space Sci. Rev., 160, 3-23, doi:10.1007/s11214-0109740-1, 2011.

Horiuchi, R. and Sato, T.: Particle simulation study of collisionless driven reconnection in a collisionless plasma, Phys. Plasmas, 1, 3587-3597, 1994.

Horiuchi, R. and Sato, T.: Particle simulation study of collisionless driven reconnection in a sheared magnetic field, Phys. Plasmas, 4, 277-289, 1997.

Ishizawa, A. and Horiuchi, R.: Suppression of Hall-Term Effects by Gyroviscous Cancellation in Steady Collisionless Magnetic Reconnection, Phys. Rev. Lett., 95, 045003, doi:10.1103/PhysRevLett.95.045003, 2005.

Karimabadi, H., Daughton, W., and Scudder, J.: Multi-scale structure of the electron diffusion region, Geophys. Res. Lett., 34, L13104, doi:10.1029/2007GL030306, 2007.

Kuznetsova, M. M., Hesse, M., and Winske, D.: Kinetic quasiviscous and bulk flow inertia effects in collisionless magnetic reconnection, J. Geophys. Res., 103, 199-213, 1998.

Lapenta, G., Markidis, S., Goldman, M. V., and Neuman, D. L.: Secondary reconnection site in reconnection-generated flux ropes and reconnection fronts, Nature Phys., 11, 690-695, doi:10.1038/nphys3406, 2015.

Nagai, T., Shinohara, I., Fujimoto, M., Matsuoka, A., Saito, Y., and Mukai, T.: Construction of magnetic reconnection in the nearEarth magnetotail with Geotail, J. Geophys. Res., 116, A04222, doi:10.1029/2010JA016283, 2011.

Nagai, T., Shinohara, I., Fujimoto, M., Matsuoka, A., Saito, Y., and Mukai, T.: Construction of magnetic reconnection in the nearEarth magnetotail with Geotail, J. Geophys. Res., 116, A04222, doi:10.1029/2010JA016283, 2011.

Nagai, T., Shinohara, I., Zenitani, S., Nakamura, R., Nakamura, T. K. M., Fujimoto, M., Saito, Y., and Mukai, T.: Threedimensional structure of magnetic reconnection in the magnetotail from Geotail observations, J. Geophys. Res., 118, 16671678, doi:10.1002/jgra.50247, 2013.

Nakamura, T. K. M., Daughton, W., Karimabadi, H., and Eriksson, S.: Three-dimensional dynamics of vortex-induced reconnection and comparison with THEMIS observations, J. Geophys. Res., 118, 5742-5757, doi:10.1002/jgra.50547, 2013.

Ng, J., Egedal, J., Le, A., and Daughton, W.: Phase space structure of the electron diffusion region in reconnection with weak guide fields, Phys. Plasmas, 19, 112108, doi:10.1063/1.4766895, 2012.

Paschmann, G., Øieroset, M., and Phan, T. D.: Geospace Environmental Modeling magnetic reconnection challenge: Simulations with a full particle electromagnetic code, Space Sci. Rev., 178, 385-417, doi:10.1007/s11214-012-9957-2, 2013.

Pritchett, P. L.: Geospace Environmental Modeling magnetic reconnection challenge: Simulations with a full particle electromagnetic code, J. Geophys. Res., 106, 3783-3798, 2011.

Ricci, P., Lapenta, G., and Brackbill, J. U.: GEM reconnection challenge: Implicit kinetic simulations with the physical mass ratio, Geophys. Res. Lett., 29, 2088, doi:10.1029/2002GL015314, 2002.

Ricci, P., Brackbill, J. U., Daughton, W., and Lapenta, G.: Collisionless magnetic reconnection in the presence of a guide field, Phys. Plasmas, 11 4102, doi:10.1063/1.1768552, 2004. 
Shay, M. A., Drake, J. F., Denton, R. E., and Biskamp, D.: Structure of the dissipation region during collisionless magnetic reconnection, J. Geophys. Res., 103, 9165-9176, 1998.

Shay, M. A., Drake, J. F., Rogers, B. N., and Denton, R. E.: Alfvénic collisionless magnetic reconnection and the Hall term, J. Geophys. Res., 106, 3759-3772, doi:10.1029/1999JA001007, 2001.

Shay, M. A., Drake, J. F., and Swisdak, M.: Two-Scale Structure of the Electron Dissipation Region during Collisionless Magnetic Reconnection, Phys. Rev. Lett., 99, 155002, doi:10.1103/PhysRevLett.99.155002, 2007.

Shuster, J. R., Chen, L.-J., Hesse, M., Argall, M. R., Daughton, W., Torbert, R. B., and Bessho, N.: Spatiotemporal evolution of electron characteristics in the electron diffusion region of magnetic reconnection: Implications for acceleration and heating, Geophys. Res. Lett., 42, 2586, doi:10.1002/2015GL063601, 2015.

Swisdak, M., Rogers, B. N., Drake, J. F., and Shay, M. A.: Diamagnetic suppression of component magnetic reconnection at the magnetopause, J. Geophys. Res., 108, 1218, doi:10.1029/2002JA009726, 2003.
Swisdak, M., Drake, J. F., Shay, M. A., and McIlhargey, J. G.: Transition from antiparallel to component magnetic reconnection, J. Geophys. Res., 110, A05210, doi:10.1029/2004JA010748, 2005.

Treumann, R. A. and Baumjohann, W.: Spontaneous magnetic reconnection: Collisionless reconnection and its potential astrophysical relevance, Astron. Astrophys. Rev., 23, 1-91, doi:101007/s00159-015-0087-1, 2015.

Wang, L., Hakim, A. H., Bhattacharjee, A., and Germaschewski, K. Comparison of multi-fluid moment models with particle-in-cell simulations of collisionless magnetic reconnection, Phys. Plasmas, 22, 012108, doi:10.1063/1.4906063, 2015.

Yamada, M., Kulsrud, R., and Ji, H.: Magnetic reconnection, Rev. Mod. Phys., 82, 603-664, doi:10.1103/RevModPhys.82.603, 2010.

Zeiler A., Biskamp, D., Drake, J. F., Rogers, B. N., Shay, M. A., and Scholer, M.: Three-dimensional particle simulations of collisionless magnetic reconnection, J. Geophys. Res., 107, 1230, doi:10.1029/2001JA000287, 2002. 